kidney cancer in job-specific subgroups including mixing and blending, bonding and cementing, and labouring. Workers in the plastics product fabricating industry had modestly elevated rates of pancreatic and brain and nervous system cancer.

Conclusions Elevated rates of lung and breast cancer among females are consistent with other studies of women in plastics and rubber manufacturing and warrant further attention in Ontario. Results for digestive and other cancers are broadly consistent with exposure to known or suspected carcinogens in these industries and suggest new sites of potential concern.

\section{0-272 EXPOSURE TO CARBAMATE INSECTICIDES AND RISKS OF NON-HODGKIN LYMPHOMAS IN THE FRENCH AGRICULTURE AND CANCER COHORT (AGRICAN)}

${ }^{1}$ Kenza Abdelmalki, Amandine Busson, Liacine Bouaoun, Séverine Tual, Alain Monnereau, Isabelle Baldi, Pierre Lebailly, Kayo Togawa. 'International Agency for Research on Cancer, France

\subsection{6/OEM-2021-EPI.18}

Introduction Existing epidemiological studies have suggested a positive association between agricultural exposure to carbamates and risks of non-Hodgkin's lymphomas (NHL); however, the association remains inconclusive with most studies lacking statistical power to examine specific carbamates and subtypes of NHL.

Objectives We estimated the associations between carbamate insecticides and the risks of NHL overall and three major histological subtypes, multiple myeloma (MM), chronic lymphocytic leukaemia/small lymphocytic lymphoma (CLL-SLL), and diffuse large B-cell lymphoma (DLBCL) in the French prospective Agriculture and Cancer cohort (AGRICAN).

Methods At enrolment (2005-2007), participants completed a questionnaire on lifetime occupational history of agricultural practices, lifestyle habits, and medical history and were followed up until 2015 through linkage to the cancer registries. Information on pesticide use for different agricultural activities (crops, animals, barns, seeds) was crossed with the French crop-exposure matrix, PESTIMAT, enabling us to assess exposure to 19 specific carbamates (e.g., carbaryl, carbofuran) by type of activity. We estimated hazard ratios (HRs) with 95\% confidence intervals (CIs) for NHL overall and its subtypes in relation to activity-specific exposure to carbamates (ever/never, duration) by fitting multivariate Cox proportional hazards models with age as the time scale. Non-users of pesticides were chosen as the reference group.

Results During the follow-up (median $=8.9$ years), 533 incident cases of NHL $(\mathrm{MM}=125, \mathrm{CLL}-\mathrm{SLL}=134, \mathrm{DLBCL}=72)$ were diagnosed. Ever use of carbaryl on animals $(\mathrm{HR}=1.84,95 \%$ $\mathrm{CI}=1.05-3.24)$ or barns $(\mathrm{HR}=1.86,95 \% \mathrm{CI}=1.06-3.27)$ were significantly associated with an increased risk of $\mathrm{MM}$, but not for crops $(\mathrm{HR}=1.24,95 \% \mathrm{CI}=0.70-2.19)$. No significant trend by exposure duration were observed. None of the other carbamate insecticides were significantly associated with the risk of MM, CLL, DLBCL or NHL overall.

Conclusion Use of carbaryl on livestock production may lead to an increased risk of MM. Studies on activity-specific exposure pathways and levels are warranted to better understand the observed association.

\section{0-276 DIESEL EXHAUST EXPOSURE AND SOMATIC MUTATIONS IN BLADDER TUMORS}

'Stella Koutros, Nicole Gonzalez, Nina Rao, Michael Dean, Donghyuk Lee, Amber Hurson, Dalsu Baris, Molly Schwenn, Ludmila Prokunina-Olsson, Melissa Friesen, Bin Zhu, Nathaniel Rothman, Debra Silverman, Alison Johnson. 'U.S. National Cancer Institute, United States

\subsection{6/OEM-2021-EPI.19}

Introduction Occupational diesel exhaust exposure has been linked to increased bladder cancer risk in epidemiologic studies, but few explore mechanistic links.

Objectives We examined the relationship between diesel exhaust exposure and somatic mutations and mutational signatures in bladder tumors.

Methods Targeted sequencing was conducted in bladder tumors from the New England Bladder Cancer Study (NEBCS), a population-based case-control study. Using data on 797 cases and 1,418 controls, two-stage polytomous logistic regression was used to calculate odds ratios (ORs) and 95\% confidence intervals (CIs) to evaluate etiologic heterogeneity between bladder cancer subtypes and quantitative estimates of respirable elemental carbon. Poisson regression was used to evaluate associations between REC and mutational signatures including those from nitro-polycyclic aromatic hydrocarbons (nitro-PAHs) in the NEBCS and in publicly available wholegenome sequencing bladder cancer dataset from The Cancer Genome Atlas Project (TCGA) (N=412).

Results We observed significant heterogeneity in the dieselbladder cancer risk relationship, with a strong positive association predominantly among cases with high-grade, non-muscle invasive TP53-mutated tumors compared to controls (ORTertile3vsUnexposed $=4.8,95 \% \mathrm{CI}=2.2,10.5 ;$ p-trend $\mathrm{T}$ mutations at $\mathrm{CpG}$ dinucleotides (Relative Risk (RR) $=1.47$, 95\% $\mathrm{CI}=1.38,1.57)$. In muscle-invasive tumors, we also observed a positive association between diesel exposure and the dieselassociated nitro-PAH signatures of 1,6-dintropyrene $(\mathrm{RR}=1.93$, $95 \% \mathrm{CI}=1.28,2.92)$ and 3 -nitrobenzoic acid $(\mathrm{RR}=1.97,95 \%$ $\mathrm{CI}=1.33,2.92)$ in pooled analyses of NEBCS and TCGA.

Conclusion The relationship between diesel exhaust and bladder cancer was heterogeneous based on the presence of TP53 mutations in tumors. Assessing the impact of diesel exhaust exposure at $\mathrm{CpG}$ dinucleotides and any resultant transcriptional changes in bladder tumors may be valuable considering the wealth of data linking particulate exposures to altered DNA methylation. Future studies that can identify nitro-PAH signatures in tumors are of interest to support the findings generated here.

\section{0-324 INCIDENCE OF POTENTIALLY EXPOSURE-RELATED CANCER SITES AMONG NORWEGIAN FIREFIGHTERS: 58 YEARS OF FOLLOW-UP}

${ }^{1}$ Niki Marjerrison, Jarle Jakobsen, Tom K Grimsrud, Johnni Hansen, Jan Ivar Martinsen, KarlChristian Nordby, Raymond Olsen, Marit B Veierød, Kristina Kjaerheim. 'Cancer Registry of Norway, Norway

\subsection{6/OEM-2021-EPI.20}

Introduction Firefighters are exposed to a variety of known and suspected carcinogens through their work, and previous studies have found elevated risk for a number of cancer sites among firefighters. 